This item was submitted to Loughborough's Institutional Repository (https://dspace.lboro.ac.uk/) by the author and is made available under the following Creative Commons Licence conditions.

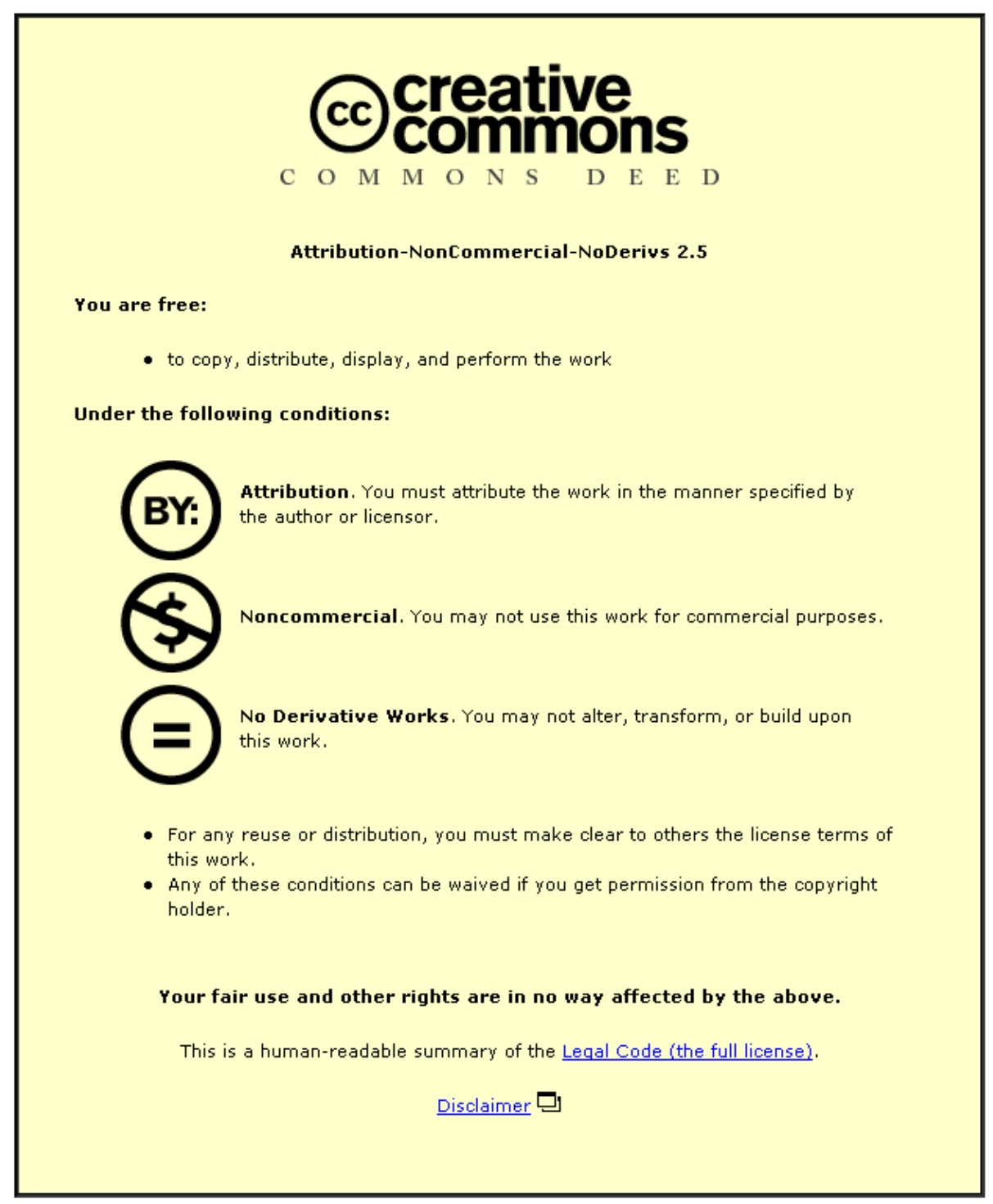

For the full text of this licence, please go to: http://creativecommons.org/licenses/by-nc-nd/2.5/ 


\title{
A Two-Step Multiuser Detection Scheme for Space-Time Coded MIMO OFDM Systems
}

\author{
Li Zhang, Mathini Sellathurai and Jonathon A. Chambers \\ Centre of Digital Signal Processing, Cardiff University \\ Cardiff CF24 0YF, UK \\ Email: \{ZhangL10,SellathuraiM,ChambersJ\}@cf.ac.uk
}

\begin{abstract}
In this paper, we investigate transceiver design for a wideband multiuser-multiple-input, multiple-output (MIMO) communication system, where the co-channel users are equipped with multiple transmit and multiple receive antennas. In particular, we develop a two-step hard-decision interference cancellation receiver for a multiuser-MIMO uplink system which employs orthogonal frequency division multiplexing (OFDM) modulation and space-time block codes (STBC). The STBC has been implemented either over adjacent tones or adjacent OFDM symbols and the performances of both implementations have been tested under slowly time-varying channels. The two-step receiver structure has been implemented using a combined interference suppression scheme based on minimum mean-squared error (MMSE) and symbol-wise likelihood detectors, which is then followed by an interference cancellation step. The receiver can suppress and cancel the interference from the co-channel users effectively without increasing the complexity significantly. The paper also includes computer experiments that are intended to improve the understanding of specific issues involved in the design of multiuser STBC-OFDM systems.
\end{abstract}

\section{INTRODUCTION}

Recently, there has been significant growth in wireless communications. The demand for multimedia services with high data rates in a multiuser communication platform is the major challenge of the current industry. In particular, spectrally efficient techniques such as OFDM, MIMO and co-channel multiuser communications are key physical layer research targets. Traditionally, power-bandwidth product has been a barrier for high speed wireless communications due to the fundamental limits on the multipath wireless channel. The introduction of MIMO techniques in the late 1990's has renewed the interest in multipath channel capacity: that is, for a system with $n$ transmit antennas and $m$ receive antennas the capacity can be increased linearly with $\min (n, m)$ without increasing the power or the bandwidth. Although MIMO pointto-point communication has attracted considerable research interest during the last decade, only recently has multiuser MIMO received attention.

On the other hand, OFDM is a very popular and robust technique that can mitigate the effect of delay spread using its ability to transform frequency selective fading channels into multiple flat fading sub channels with the help of a cyclic prefix, and can be effectively combined with the space-time block codes originally designed for flat fading channels. In practice, OFDM may be used in combination with space-time block codes and the space-time block coding can either be implemented over adjacent tones or adjacent OFDM symbols. However, the performance of such STBC-OFDM systems may seriously degrade in the presence of multiple-access interference (MAI) resulting from multiuser communications when multiple users are allowed to access the same channel simultaneously. Therefore, it is of importance to design a system receiver that can suppress co-channel interference by including a suitable multiuser detection scheme and effective channel equalizer.

In this paper, we consider a wide-band uplink system with $K$ synchronous co-channel users, each is equipped with Alamouti's space-time block coded (STBC) and OFDM modulated transmit antennas (use the same scheme as [1] and [2]). In OFDM modulation, assuming that the channel remains constant over continuous tones of each block, we propose to employ the STBC over the adjacent tones, which is suitable for slow fading environments with large delay spreads. The number of transmit antennas per user is limited to $n_{t}=2$. The receiving station is equipped with $m(\geq K)$ receive antennas. The challenge is to design a receiver that can suppress the co-channel interferences and inter-symbol interferences simultaneously. We extend the narrow band two-step interference suppression and cancellation scheme proposed in [2] to the proposed wideband channels.

Note that the classical MMSE interference suppression technique has been proposed in [3] for multiuser STBCOFDM systems. The proposed two-step interference cancellation receiver brings considerable performance gain while maintaining decoding simplicity and without increasing the complexity of the receiver considerably compared to classical MMSE receivers. Moreover, [3] employs STBC over two OFDM symbols assuming that the channel is constant over the adjacent OFDM symbols and its sensitivity to the channel variation within the two symbol period is significantly higher than the proposed transmitter. Further, the iterative interference cancellation receiver schemes for the proposed scenario may achieve better performance but with considerable complexity increase (see in [7]).

Notation: Bold upper case $\mathbf{X}$ denotes a matrix and lowercase $\mathbf{x}$ denotes a vector. $\mathbf{X}^{(i)}$ and $\mathbf{x}^{(i)}$ denote the signal matrix and vector corresponding to the $i$ th user. We use $x(k)$ to denote the $k$ th element of the vector $\mathbf{x}$ of size $N$, where $k=0,1, \ldots N-1 . \mathbf{x}_{n}$ denotes the $n$th block vector in the data stream. The matrix indexed by $q$ and $j$ is denoted by $\mathbf{X}_{q j}$. 


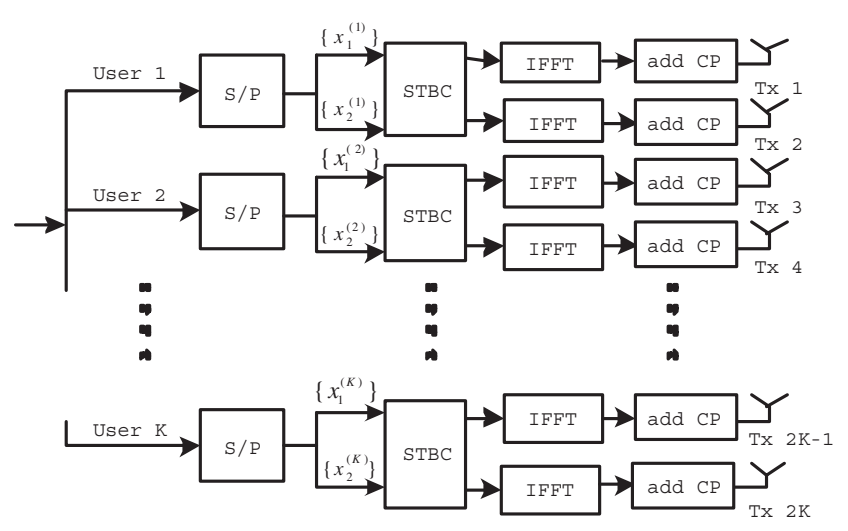

Fig. 1. Baseband Multiuser STBC MIMO-OFDM transmitter.

$\mathbf{I}_{N}$ is an identity matrix of size $N$. Complex conjugation, transposition and conjugate transposition of a matrix are respectively denoted by $(\cdot)^{*},(\cdot)^{T}$ and $(\cdot)^{H}$.

\section{SYSTEM MODEL}

We consider a $K$-user space-time block coded OFDM wireless communication system as illustrated in Fig 1, where each user terminal is equipped with $n_{t}=2$ transmit antennas. First, the incoming data stream is space-time block encoded and then an OFDM modulator is applied to the outputs of the space time encoder. The outputs of the OFDM modulators after addition of cyclic prefix bits are transmitted using multiple antennas simultaneously.

Here, there are two popular ways to extend space-time block codes combined with OFDM modulation, which are traditionally designed for single-tone flat fading channels, to multipletone OFDM modulation: (i) STBC is performed with the time index replaced by the tone index in OFDM. This requires that the channel remains constant over a number of consecutive tones equal to the number of transmit antennas $n_{t}$ (see in [3]); (ii) STBC on a per-tone basis across OFDM symbols in time exactly as performed in the flat fading environments [1]. However, this requires that the channel remains constant over $n_{t}$ OFDM symbol period which is equal to the duration of $n_{t}(N+L-1) / B$, where $L$ denotes frequency-selective subchannel tap length and $B$ denotes transmission bandwidth.

\section{A. STBC over OFDM symbols for $n_{t}=2$}

Consider two consecutive transmitting OFDM symbol blocks $\mathbf{x}_{1}^{(i)}=\left[x_{1}^{(i)}(0), \cdots, x_{1}^{(i)}(N-1)\right]^{T}$ and $\mathbf{x}_{2}^{(i)}=$ $\left[x_{2}^{(i)}(0), \cdots, x_{2}^{(i)}(N-1)\right]^{T}$, which are the frequency domain symbols from the $i$ th user, $i=1, \ldots, K$. The output of the STBC encoder is converted into the time domain samples by N-point inverse DFT operation $\mathbf{F}^{H}$, where the $\mathbf{F}$ denotes the $N \times N$ DFT matrix whose $m n$th $(m, n=1,2, \ldots N)$ element is given by $\mathbf{F}=\frac{1}{\sqrt{N}} e^{-j 2 \pi(m-1)(n-1) / N}$ [6]. Consequently, the OFDM modulated STBC coded time-domain symbols of the $i$ th user during two consecutive block intervals can be written in the matrix form: $\left[\begin{array}{cc}\mathbf{F}^{H} \mathbf{x}_{1}^{(i)} & \mathbf{F}^{H} \mathbf{x}_{2}^{(i)} \\ -\mathbf{F}^{H} \mathbf{x}_{2}^{(i)^{*}} & \mathbf{F}^{H} \mathbf{x}_{1}^{(i)^{*}}\end{array}\right]$. At a given block interval, $\mathbf{F}^{H} \mathbf{x}_{1}^{(i)}$, and $\mathbf{F}^{H} \mathbf{x}_{2}^{(i)}$ denote the signal blocks transmitted by antenna one and two after OFDM modulation, respectively, without cyclic prefix of course. Then, during the next block interval $-\mathbf{F}^{H} \mathbf{x}_{2}^{(i)^{*}}$ and $\mathbf{F}^{H} \mathbf{x}_{1}^{(i)^{*}}$ are respectively sent by antenna one, and two.

At the receiving end, after FFT operation and cyclic prefix removal, the $N$ received symbols in the frequency domain collected from the $j$ th receive antenna during the first and second OFDM intervals can be represented as

$$
\begin{gathered}
\mathbf{r}_{1 j}=\sum_{i=1}^{K}\left\{\mathbf{F}\left[\mathbf{H}_{c}^{(i)}\right]_{1 j} \mathbf{F}^{H} \mathbf{x}_{1}^{(i)}+\mathbf{F}\left[\mathbf{H}_{c}^{(i)}\right]_{2 j} \mathbf{F}^{H} \mathbf{x}_{2}^{(i)}\right\}+\mathbf{v}_{1 j} \\
\mathbf{r}_{2 j}=\sum_{i=1}^{K}\left\{\mathbf{F}\left[\mathbf{H}_{c}^{(i)}\right]_{2 j} \mathbf{F}^{H} \mathbf{x}_{1}^{(i)}-\mathbf{F}\left[\mathbf{H}_{c}^{(i)}\right]_{1 j} \mathbf{F}^{H} \mathbf{x}_{2}^{(i)^{*}}\right\}+\mathbf{v}_{2 j}
\end{gathered}
$$

where $\mathbf{v}_{1 j}$ and $\mathbf{v}_{2 j}$ are the frequency response vectors of zero mean complex white Gaussian noise which influences on the $j$ th receive antenna; $\left[\mathbf{H}_{c}^{(i)}\right]_{q j}$ is the channel impulse response circular convolution matrix of size $N \times N$ which is experienced by the $i$ th user's signal transmitted from the $q$ th transmit antenna to the $j$ th receive antenna, and $q \in\{1,2\}$, (see in [5]), Note that, it is assumed that the channel responses are constant during two consecutive signal block intervals, i.e. quasi-static.

We define the subcarrier coupling matrix $\mathbf{H}_{q j}^{(i)}$ for the equivalent frequency response of the channel as $\mathbf{H}_{q j}^{(i)}=\mathbf{F}\left[\mathbf{H}_{c}^{(i)}\right]_{q j} \mathbf{F}^{H}$. Because we consider the transmission over time-invariant frequency-selective fading channels, the the equivalent frequency channel response is represented in a diagonal matrix form as $\mathbf{H}_{q j}^{(i)}=\operatorname{diag}\left[H_{q j}^{i}(0), H_{q j}^{i}(1), \ldots, H_{q j}^{i}(N-1)\right]$. Hence, let $H_{1 j}^{i}(k)$ and $H_{2 j}^{i}(k)$ denote the channel frequency response of the $k$ th tone, corresponding to the channel from the $1 s t$ and $2 n d$ transmit antennas of the $i$ th user to the $j$ th receive antenna, respectively, over two continuous signal block intervals. Due to employing space-time block encoding scheme, the $k$ th tone receive symbol in frequency domain over the two sequential OFDM time-slots 1 and 2 could be obtained in (3) and (4), respectively, where $k=0,1, \ldots, N-1$,

$$
\begin{gathered}
r_{1 j}(k)=\sum_{i=1}^{K}\left\{H_{1 j}^{i}(k) x_{1}^{(i)}(k)+H_{2 j}^{i}(k) x_{2}^{(i)}(k)\right\}+v_{1 j}(k) \\
r_{2 j}(k)=\sum_{i=1}^{K}\left\{H_{2 j}^{i}(k) x_{1}^{(i)^{*}}(k)-H_{1 j}^{i}(k) x_{2}^{(i)^{*}}(k)\right\}+v_{2 j}(k)
\end{gathered}
$$

where $\left[v_{1 j}(k) v_{2 j}(k)\right]$ represent the frequency domain representation of the receiver AWGN at the $k$ th tone. We next define $\mathbf{r}_{j}(k)=\left[\begin{array}{ll}r_{1 j}(k) & r_{2 j}^{*}(k)\end{array}\right]^{T}, \mathbf{x}^{(i)}(k)=\left[x_{1}^{(i)}(k) x_{2}^{(i)}(k)\right]^{T}, \mathbf{v}_{j}(k)=$ $\left[v_{1 j}(k) v_{2 j}^{*}(k)\right]^{T}$ and $\breve{\mathbf{H}}_{j}^{i}=\left[\begin{array}{cc}H_{1 j}^{i}(k) & H_{2 j}^{i}(k) \\ H_{2 j}^{i}(k) & -H_{1 j}^{i}{ }^{*}(k)\end{array}\right]$, where $\breve{\mathbf{H}}_{j}^{i}$ is the equivalent channel response matrix of the $k$ th tone between the $i$ th user terminal and the $j$ th receive antenna. The $k$ th tone receive signals from all receive antennas during two consecutive OFDM periods can be represented in matrix form as follows:

$$
\left[\begin{array}{c}
\mathbf{r}_{1}(k) \\
\mathbf{r}_{2}(k) \\
\vdots \\
\mathbf{r}_{m}(k)
\end{array}\right]=\left[\begin{array}{ccc}
\breve{\mathbf{H}}_{1}^{1}(k) & \cdots & \breve{\mathbf{H}}_{1}^{K}(k) \\
\breve{\mathbf{H}}_{2}^{1}(k) & \cdots & \breve{\mathbf{H}}_{2}^{K}(k) \\
\vdots & \ddots & \vdots \\
\breve{\mathbf{H}}_{m}^{1}(k) & \cdots & \breve{\mathbf{H}}_{m}^{K}(k)
\end{array}\right]\left[\begin{array}{c}
\mathbf{x}^{(1)}(k) \\
\mathbf{x}^{(2)}(k) \\
\vdots \\
\mathbf{x}^{(K)}(k)
\end{array}\right]+\left[\begin{array}{c}
\mathbf{v}_{1}(k) \\
\mathbf{v}_{2}(k) \\
\vdots \\
\mathbf{v}_{m}(k)
\end{array}\right]
$$


We re-write the receive signal for simplicity of notation, as follows:

$$
\tilde{\mathbf{r}}(k)=\tilde{\mathbf{H}}(k) \tilde{\mathbf{x}}(k)+\mathbf{v}(k)
$$

where $\tilde{\mathbf{r}}(k)$ is the overall receive signal vector at all receive antennas with $2 m$ elements, $\tilde{\mathbf{H}}(k)$ is the equivalent overall channel response matrix of size $2 m \times 2 K, \tilde{\mathbf{x}}(k)$ is the transmitting signal vector with $2 K$ elements including all users at the $k$ th tone, and $\mathbf{v}(k)$ is the equivalent complex noise vector of size $2 m$ which influences the overall channels.

\section{B. STBC over OFDM carriers for $n_{t}=2$}

Alamouti's space-time block encoding can also be done across two consecutive tones $k$ and $k+1$. In this context, we assume $H_{q j}(k)=H_{q j}(k+1)$. If the transmitting signal from the $i$ th user is defined as $\mathbf{x}^{(i)}=\left[x^{(i)}(0), \cdots, x^{(i)}(N-1)\right]^{T}$, then, the coded signal from two transmit antennas of the $i$ th user during two consecutive transmitting symbols could be represented in a matrix form as $\left[\begin{array}{cc}x^{(i)}(k) & x^{(i)}(k+1) \\ -x^{(i)^{*}}(k+1) & x^{(i)^{*}}(k)\end{array}\right]$. The two symbols $x_{1}^{(i)}$ and $x_{2}^{(i)}$ are transmitted over antennas 1 and 2 respectively on tone $k$ and $-x_{2}^{(i)^{*}}$ and $x_{1}^{(i)^{*}}$ are transmitted over antennas 1 and 2 respectively on tone $k+1$ within the same OFDM symbol.

The receive signal at the $j$ th receive antenna during two sequential symbol times will be given by,

$$
\begin{gathered}
r_{j}(k)=\sum_{i=1}^{K}\left\{H_{1 j}^{i}(k) \cdot x^{(i)}(k)+H_{2 j}^{i}(k) \cdot x^{(i)}(k+1)\right\}+v_{j}(k) \\
r_{j}(k+1)=\sum_{i=1}^{K}\left\{\begin{array}{c}
H_{2 j}^{i}(k+1) \cdot x^{(i)^{*}}(k) \\
-H_{1 j}^{i}(k+1) \cdot x^{(i)^{*}}(k+1)
\end{array}\right\}+v_{j}(k+1)
\end{gathered}
$$

\section{Two STEP MMSE INTERFERENCE CANCELLATION}

We extend the two-step generalized MMSE interference receiver in [2] to the proposed multiuser STBC-OFDM. For simplicity, we consider only the two-user scenario. The receiver structure is illustrated in Fig 2.

In this receiver structure, the OFDM-demodulated received signal will be passed through the following three parts of processing:

(1) Signal estimation based on a combined MMSE and multiuser detector;

(2) Multiple-access Interference cancellation and symbol estimation use the modified interference-free received signal;

(3)Decision based on the reliability estimation.

\section{A. Step 1: Linear MMSE Interference Suppression and Symbol Estimation}

We use an MMSE interference cancellation and symbolwise likelihood decoder to estimate the transmitted symbols by the users. Consider the $i$ th user terminal, where $i=1,2$. We define the following cost function to minimize the mean squared error caused by co-channel interference and noise in the symbol $\hat{x}^{(i)}$, where $\hat{x}^{(i)}=\mathbf{w} \tilde{\mathbf{r}}$ is the estimation of the symbol $x^{(i)}: J(\mathbf{w})=E\left\{\left\|x^{(i)}-\mathbf{w} \tilde{\mathbf{r}}(k)\right\|^{2}\right\}$, where $E\{\cdot\}$ denotes the statistical expectation operator. To minimize the mean squared error, we choose the weight vector $\mathbf{w}$ of size $2 m$ based on standard minimization techniques: $\frac{\partial J(\mathbf{w})}{\partial \mathbf{w}}=0$. Hence, the weight vectors $\mathbf{w}_{2 i-1}=\left[w_{2 i-1,1}, w_{2 i-1,2}, \ldots, w_{2 i-1,2 m}\right]$ and $\mathbf{w}_{2 i}=\left[w_{2 i, 1}, w_{2 i, 2}, \ldots, w_{2 i, 2 m}\right]$, corresponding to the $i$ th user, can be computed respectively, as

$$
\begin{aligned}
\mathbf{w}_{2 i-1} & =\mathbf{M}^{-1} \tilde{\mathbf{h}}_{2 i-1} \\
\mathbf{w}_{2 i} & =\mathbf{M}^{-1} \tilde{\mathbf{h}}_{2 i}
\end{aligned}
$$

where $\mathbf{M}=\tilde{\mathbf{H}}(k) \tilde{\mathbf{H}}^{H}(k)+\frac{1}{\tau} \mathbf{I}_{2 m}$ and $\tau=E_{s} / N_{0}$ is the signal to noise ratio, $\tilde{\mathbf{h}}_{2 i-1}$ and $\tilde{\mathbf{h}}_{2 i}$ are the $(2 i-1)$ th and $(2 i)$ th columns of $\tilde{\mathbf{H}}(k)$, respectively. Then, the symbol-wise likelihood decoding equation can be written as

$$
\hat{\mathbf{x}}^{(i)}(k)=\arg \min _{\left[\hat{x}_{1}^{(i)} \hat{x}_{2}^{(i)}\right] \in \mathbf{X}}\left\{\begin{array}{c}
\left\|\mathbf{w}_{2 i-1}^{*} \tilde{\mathbf{r}}(k)-\hat{x}_{1}^{(i)}(k)\right\|_{2}^{2} \\
+\left\|\mathbf{w}_{2 i}^{*} \tilde{\mathbf{r}}(k)-\hat{x}_{2}^{(i)}(k)\right\|^{2}
\end{array}\right\}
$$

where $\left[\hat{x}_{1}^{(i)} \hat{x}_{2}^{(i)}\right]$ includes all possible symbol pairs in the transmitted signal. Both $\hat{x}_{1}^{(i)}(k)$ and $\hat{x}_{2}^{(i)}(k)$ are scalar signal symbols at the $k$ th tone, which can be decided independently, because the metrics separate easily.

Finally, the reliability function for the first step estimation of the symbol from the $i$ th user terminal can be represented as

$$
\triangle_{\hat{\mathbf{x}}^{(i)}(k)}=\left\|\mathbf{w}_{2 i-1}^{*} \tilde{\mathbf{r}}(k)-\hat{x}_{1}^{(i)}(k)\right\|^{2}+\left\|\mathbf{w}_{2 i}^{*} \tilde{\mathbf{r}}(k)-\hat{x}_{2}^{(i)}(k)\right\|^{2}
$$

\section{B. Step 2: Two-Step Approach for MAI Cancellation and Symbol Re-estimation}

In Step 1, a linear MMSE scheme is used to suppress the interferences from the co-channel users in estimating the transmitted information symbols. In the second step, we reestimate the transmitted symbols based on a modified received signal obtained by cancelling the multiple access interferences. Note that a perfect interference cancellation may be achieved only if the signals from the first step have been decoded correctly. In the case of two users, in order to estimate the second user symbols, the receiver cancels the multiple access interference (MAI) caused by the first user from the received signal $\mathbf{r}_{1}(k), \mathbf{r}_{2}(k), \ldots, \mathbf{r}_{m}(k)$ to estimate the transmitted symbols. For the $k$ th tone, the modified received signal vectors after cancelling the MAI from the first user will be represented as

$$
\begin{gathered}
\mathbf{a}_{1}(k)=\mathbf{r}_{1}(k)-\breve{\mathbf{H}}_{1}^{1}(k) \hat{\mathbf{x}}^{(1)}(k) \\
\mathbf{a}_{2}(k)=\mathbf{r}_{2}(k)-\breve{\mathbf{H}}_{2}^{1}(k) \hat{\mathbf{x}}^{(1)}(k) \\
\ldots \\
\ldots \\
\mathbf{a}_{m}(k)=\mathbf{r}_{m}(k)-\breve{\mathbf{H}}_{m}^{1}(k) \hat{\mathbf{x}}^{(1)}(k)
\end{gathered}
$$

The user 2's $k$ th signals are re-decoded using the above modified received vector:

$$
\hat{\hat{\mathbf{x}}}^{(2)}(k)=\arg \min _{\hat{\hat{\mathbf{x}}}(2)} \sum_{\mathbf{x}}^{m}\left\{\left\|\mathbf{a}_{n}-\breve{\mathbf{H}}_{n}^{2}(k) \hat{\hat{\mathbf{x}}}^{(2)}(k)\right\|^{2}\right\}
$$




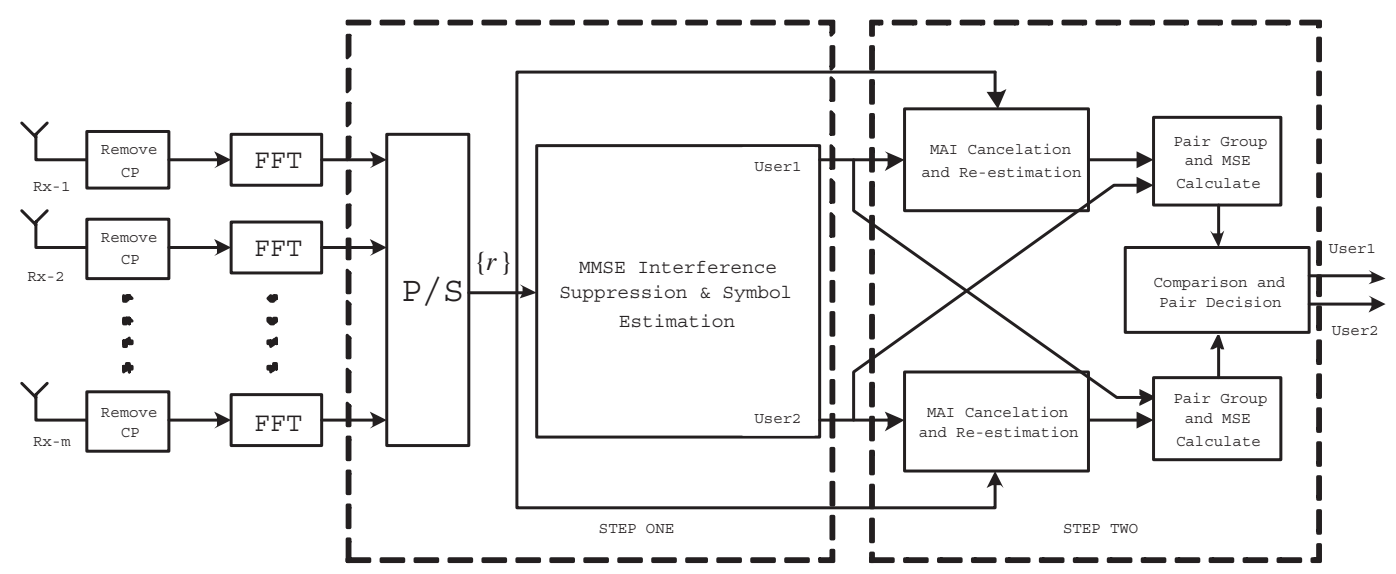

Fig. 2. Two-step STBC MIMO-OFDM receiver for two users

where $\mathbf{x}$ includes all possible choices of QPSK constellations. The reliability function for the estimation from this second step of the user 2 will be given by

$$
\triangle_{\hat{\mathbf{x}}^{(2)}(k)}=\sum_{n=1}^{m}\left\{\left\|\mathbf{a}_{n}-\breve{\mathbf{H}}_{n}^{2}(k) \hat{\hat{\mathbf{x}}}^{(2)}(k)\right\|^{2}\right\}
$$

Finally, the total reliability of the first and second step estimation will be the summation of the reliability of the first user at the first step and the reliability of the second user at the second step, which is given by the overall reliability $\triangle_{a}=\triangle_{\hat{\mathbf{x}}^{(1)}(k)}+\triangle_{\hat{\mathbf{x}}^{(2)}(k)}$ for $\hat{\mathbf{x}}^{(1)}(k)$ and $\hat{\hat{\mathbf{x}}}^{(2)}(k)$. This procedure will be repeated assuming correct decision of the second user at the first step and based upon its estimate, the user 1 signals will be re-estimated.

At the next step, the receiver then repeats the MAI interference cancellation and symbol-wise likelihood re-estimation algorithm, assuming that the estimation of the second user transmitted signal at the $k$ th tone $\hat{\mathbf{x}}^{(2)}(k)=\left[\hat{x}_{1}^{(2)}(k), \hat{x}_{2}^{(2)}(k)\right]^{T}$ has been decoded correctly at the first step. In a similar fashion, we can also define the reliability function as $\triangle_{b}=$ $\triangle_{\hat{\mathbf{x}}^{(2)}(k)}+\triangle_{\hat{\mathbf{\mathbf { x }}}^{(1)}(k)}$ to denote the overall reliability for $\hat{\mathbf{x}}^{(2)}(k)$ and $\hat{\mathbf{x}}^{(1)}(k)$.

Finally, the symbol pairs based on the best overall reliabilities obtained between $\Delta_{a}$ and $\Delta_{b}$ will be selected. When $\triangle_{a}<\triangle_{b}$, the pair $\left[\hat{\mathbf{x}}^{(1)}(k), \hat{\hat{\mathbf{x}}}^{(2)}(k)\right]$ will be chosen as the estimates, otherwise, it is $\left[\hat{\mathbf{x}}^{(1)}(k), \hat{\mathbf{x}}^{(2)}(k)\right]$.

\section{Algorithm Complexity Discussion}

Following the discussion in section II, the proposed algorithm is only based on Linear MMSE and simple symbol-wise likelihood detection. Therefore, the symbols can be estimated with much lower computational complexity as compared with the alternative Multiuser receiver in [6]. The dimensions of matrix $\tilde{\mathbf{H}}$ are $2 m \times 2 K$. In each linear MMSE cancellation, to find the equalizer coefficient weight vector $\mathbf{w}$, the algorithm requires the inversion of $\left[\tilde{\mathbf{H}} \cdot \tilde{\mathbf{H}}^{H}+\frac{1}{\tau} \mathbf{I}_{2 m}\right]$ that needs $O\left(4 m^{2}\right)$ operations and must be performed $N$ times for estimation of each signal block per user. Because of the simple computation of the other symbol-wise estimation part, the algorithm only requires $O\left(4 \mathrm{Nm}^{2}\right)$ operations in each estimation of the block. The simulation results, in section IV, show that the performance of the proposed scheme will be significantly greater than the Linear MMSE algorithm. Therefore, the two-step interference cancellation receiver brings considerable performance gain while maintaining decoding simplicity and without increasing the complexity of the receiver considerably compared to the classical MMSE receivers.

\section{SIMULATION}

In order to illustrate the performance of the proposed twostep receiver structure in principle, we run the simulations simply based on a two user space-time block coded MIMO OFDM system case equipped with two transmit antennas for each user and two receive antennas. Each user data stream contains 256 symbols, which is coded into two data blocks by space time encoding. For the tones orthogonal to each other, the assumed bandwidth of $1 \mathrm{MHz}$ baseband is divided into 128 sub-carriers by IFFT operation on each time slot with $128 \mu$ s duration. The QPSK signal modulation is employed at the transmitting stage. Moreover, an additional 22 cyclic prefix symbols are used as a guard interval after each data block. Therefore, in this case, there contains $512 \mu \mathrm{s}$ information duration and $88 \mu \mathrm{s}$ additional cyclic prefix in one transmitting signal frame totally. The data transmission is implemented simply over a MIMO frequency selective channel with slow time variant fading, generated by the typical Jakes fading model (see in [4]) and 3-delay taps have been introduced into each sub-channel, i.e. frequencyselective sub-channel tap length $L=3$ and $\sum_{l=1}^{L} \sigma_{l}^{2}=1$, where $\sigma_{l}^{2}$ is the variance of the $l$ th path. The channel fading is also simplified with assumption of uncorrelation among different transmitting antennas of different users. Otherwise, the perfect knowledge of the channel state at the receiver is assumed at any time.

Fig. 3 shows the comparison of system performance between linear MMSE interference cancellation and two-step processing when the system is tested with various fading rate channel environments. The best performance is given 


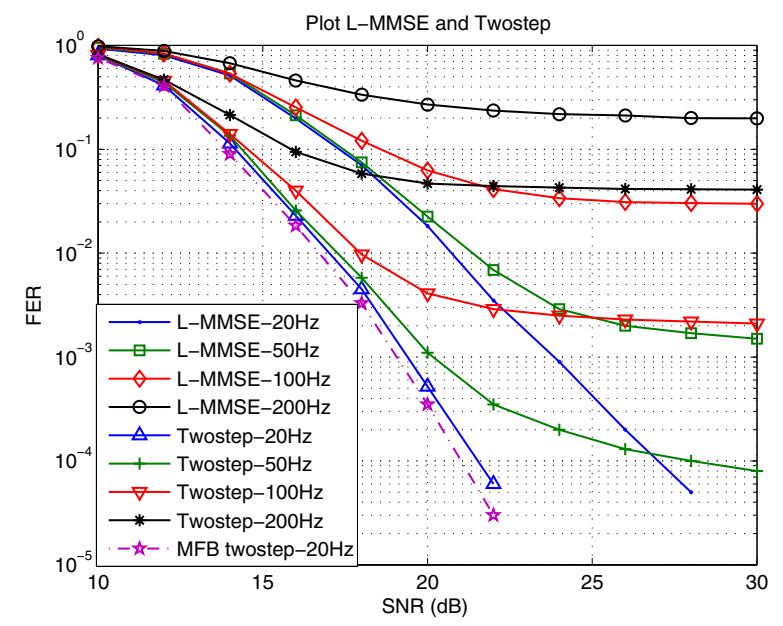

Fig. 3. Performance comparison between typical MMSE and two-step processing over various fading rates.

at the channel of $20 \mathrm{~Hz}$ maximum Doppler frequency. As a benchmark, we also evaluate performance of the matched filter for two-step processing, assuming perfect knowledge of the interfering symbols [5] at maximum Doppler frequency of $20 \mathrm{~Hz}$. The two-step interference cancellation scheme performs very close to the match filter bound (MFB), as a result, the channel variation has not influenced the receiver at slow fading rate and the ISI and MAI have been cancelled effectively. Here, we use the typical STBC scheme as in [1] in order to attain double spatial diversity compared with normal MIMO transmission. Hence, by explanation in [1], [2] and [8], this two-step scheme could achieve a diversity order of $2 \times(\mathrm{m}-$ $K+1)$. Otherwise, the performance degrades at higher SNR values with increasing maximum Doppler frequency. Recall that the channel is assumed to be quasi-static over two OFDM symbol periods in order to employ space-time block codes. This assumption will be violated in this case and the spacetime block code and OFDM fails to keep their orthogonality between the antennas and the carriers which causes for error floor.

Fig.4 shows the performance comparison between the block encoding scheme and symbol encoding scheme, as called STBC(I) and STBC(II) respectively, over various fading rate channels. The symbol encoding scheme outperforms the performance of the block encoding scheme, particularly, when the maximum Doppler frequency is high. The block STBC fails due to the channel variation between two blocks whereas the symbol encoding scheme is not affected by the channel variation between two blocks. However, for the OFDM symbols to be orthogonal, the channel should remain constant within an OFDM block, which is the reason for the error-floor in the varying channels.

\section{CONCLUSION}

In this paper, we have addressed the design of a two-step interference cancellation receiver to cancel multiple access inter-

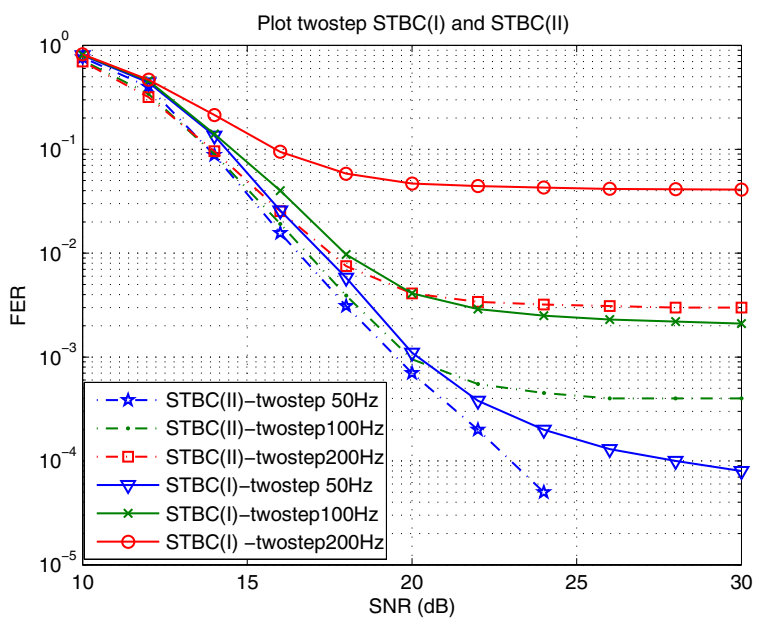

Fig. 4. Performance comparison between block encoding scheme and symbol encoding scheme.

ference (MAI) in a multiuser MIMO wideband wireless communication system within a slow fading channel environment. In this approach, the transmitter uses space-time block codes and OFDM modulation, which are serially concatenated. The receiver is based on a two-step hard-interference cancellation algorithm. The simulation results indicate that the proposed scheme could obtain substantial performance improvement without increasing the complexity compared with the Linear MMSE scheme. By concatenating the transmitter with an outer channel code, for example, turbo or low-density parity check (LDPC) code, in addition to the multiple transmit and receive antenna diversities, the frequency selective diversity of order $L$ can also be gained by the proposed receiver.

\section{REFERENCES}

[1] S. M. Alamouti, A simple transmit diversity technique for wireless communications, IEEE J. Selected Areas Communications, Volume16, issue 8, pp. 1451 - 1458, October 1998.

[2] A. F. Naguib, N. Seshadri and A. R. Calderbank, Application of spacetime coding and interference suppression for high capacity and high data rate wireless system, in proc. 32nd Asilomar Conf. Signals, System, and computer, vol.2, Pacific Grove, CA, pp. 1803 - 1810, November 1998.

[3] S. Suthaharan, A. Nallanathan and B. Kannan, Space-Time coded MIMOOFDM for high capacity and high data-rate wireless communication over frequency selective fading channels, Mobile and Wireless Communications Network 2002, 4th International Workshop, pp. 424 - 428, September 2002.

[4] P. Dent, G. E. Bottomley and T. Croft, Jakes fading model revisited, Electronics Letters, Volume 29, Issue 13, pp. 1162 - 1163, June 1993.

[5] P. Schniter, Low-complexity equalization of OFDM in doubly selective channels, Signal Processing, IEEE Transactions on Volume 52, Issue 4, pp. 1002 - 1011, April 2004.

[6] P. L. Kafle, A. B. Sesay and J. Mcrory, An iterative MMSE-decision feedback multiuser detector for space-time coded multicarrier CDMA system, Electrical and Computer Engineering, 2004. Canadian Conference, pp. 2281 - 2286 Volume 4, May 2004.

[7] A. R. Golshan and K. M. Chugg, A reduced complexity algorithm for iterative multiuser detection and decoding of asynchronous users, Communications, ICC 2001. IEEE International Conference, Volume 3, pp. 847 - 851 June 2001.

[8] E. A. Fain and M. K. Varanasi, Diversity order gain for narrowband multiuser communications with precombining group detection, IEEE Trans. Commun., volume 48, Issue 4, pp. 533 - 536, April. 2000. 